\title{
Anti-PD-1 Monoclonal Antibody Sym021
}

National Cancer Institute

\section{Source}

National Cancer Institute. Anti-PD-1 Monoclonal Antibody Sym021. NCI Thesaurus. Code C142865.

A humanized monoclonal antibody directed ag ainst the negative immunoregulatory human cell surface receptor programmed cell death 1 (PD-1, PCD-1; PDCD1), with potential immune checkpoint inhibitory and antineoplastic activities. Upon administration, anti-PD-1 monoclonal antibody Sym021 binds to and inhibits PD-1 activation and its downstream signaling pathways. This may restore immune function through the activation of T-cells and cell-mediated immune responses against tumor cells. PD-1, a transmembrane protein in the immunoglobulin superfamily (IgSF), is expressed on T-cells and functions as an immune checkpoint that negatively regulates $\mathrm{T}$-cell activation and effector function when activated by its ligands programmed cell death ligand 1 (PD-L1) or 2 (PD-L2). Activated PD-1 plays an important role in tumor evasion from host immunity. 